\title{
Assessment of Functionals for First-Principle Studies of the Structural and Electronic Properties of $\delta$ - $\mathrm{Bi}_{2} \mathrm{O}_{3}$
}

\author{
D. H. Galván, ${ }^{1}$ R. Núñez-González, ${ }^{2}$ R. Rangel, ${ }^{3}$ P. Alemany, ${ }^{4,5}$ and A. Posada-Amarillas ${ }^{6}$ \\ ${ }^{1}$ Centro de Nanociencias y Nanotecnología, Universidad Nacional Autónoma de México, Apartado Postal 2681, \\ 22800 Ensenada, BCN, Mexico \\ ${ }^{2}$ Departamento de Matemáticas, Universidad de Sonora, 83000 Hermosillo, SON, Mexico \\ ${ }^{3}$ División de Estudios de Posgrado, Facultad de Ingeniería Química, Edificio V1, Ciudad Universitaria, 58060 Morelia, MICH, Mexico \\ ${ }_{4}^{4}$ Instituto de Química Teórica i Computacional (IQTCUB), Universitat de Barcelona, Marti i Franqués 1-11, 08028 Barcelona, Spain \\ ${ }^{5}$ Departamento de Química Física, Universitat de Barcelona, Marti i Franqués 1-11, 08028 Barcelona, Spain \\ ${ }^{6}$ Departamento de Investigación en Física, Universidad de Sonora, Apartado Postal 5-088, 83190 Hermosillo, SON, Mexico
}

Correspondence should be addressed to R. Núñez-González; ronunez@gauss.mat.uson.mx

Received 19 April 2015; Revised 29 June 2015; Accepted 1 July 2015

Academic Editor: Markus R. Wagner

Copyright (C) 2015 D. H. Galván et al. This is an open access article distributed under the Creative Commons Attribution License, which permits unrestricted use, distribution, and reproduction in any medium, provided the original work is properly cited.

Fully relativistic full-potential density functional calculations with an all-electron linearized augmented plane waves plus local orbitals method were carried out to perform a comparative study on the structural and electronic properties of the cubic oxide $\delta$ - $\mathrm{Bi}_{2} \mathrm{O}_{3}$ phase, which is considered as one of the most promising materials in a variety of applications including fuel cells, sensors, and catalysts. Three different density functionals were used in our calculations, LDA, the GGA scheme in the parametrization of Perdew, Burke, and Ernzerhof (PBE96), and the hybrid scheme of Perdew-Wang B3PW91. The examined properties include lattice parameter, band structure and density of states, and charge density profiles. For this modification the three functionals reveal the characteristics of a metal and the existence of minigaps at high symmetry points of the band structure when spin-orbit coupling is taken into account. Density of states exhibits hybridization of $\mathrm{Bi} 6 \mathrm{~s}$ and O $2 \mathrm{p}$ orbitals and the calculated charge density profiles exhibit the ionic character in the chemical bonding of this compound. The B3PW91 hybrid functional provided a better agreement with the experimental result for the lattice parameter, revealing the importance of Hartree-Fock exchange in this compound.

\section{Introduction}

The VI-bismuth compounds $\mathrm{Bi}_{2} \mathrm{O}_{3}, \mathrm{Bi}_{2} \mathrm{~S}_{3}$, and $\mathrm{Bi}_{2} \mathrm{Te}_{3}$ comprise a family of technological semiconductors [1] employed in diverse areas of industry. $\mathrm{Bi}_{2} \mathrm{O}_{3}$ has been used as an effective cocatalyst for oxidation of ammonia since the beginning of the last century; also it has been used as the precursor for the production of $\mathrm{Bi}_{2} \mathrm{MoO}_{6}$, a catalyst popularly used in the selective oxidation of propylene into acrolein [2]. Galván et al. [3] showed that, using the same compound in $\mathrm{CO}$ to $\mathrm{CO}_{2}$ conversion, a significant improvement in the oxidation process could be obtained, while it is likely to be used as a catalyst in catalytic converters to clean the exhaust discharge. Moreover, metal oxides, bismuth oxides, and rareearth oxides form the basis for some of the ceramic high $T_{c^{-}}$superconductors [4].
Bismuth oxide is a polymorphic material that crystallizes in five modifications [5]. $\delta-\mathrm{Bi}_{2} \mathrm{O}_{3}$ is formed from heating monoclinic $\alpha-\mathrm{Bi}_{2} \mathrm{O}_{3}$ at $730^{\circ} \mathrm{C}$ and it is stable up to the melting point of $824^{\circ} \mathrm{C}$. Tetragonal $\left(\beta-\mathrm{Bi}_{2} \mathrm{O}_{3}\right)$ and bodycentered cubic $\left(\gamma-\mathrm{Bi}_{2} \mathrm{O}_{3}\right)$ modifications have been obtained below $650^{\circ} \mathrm{C}$ as metastable phases [6]. The hydrothermal method was used to obtain $\varepsilon-\mathrm{Bi}_{2} \mathrm{O}_{3}$ phase [7]. It is currently accepted that the cubic oxide $\delta-\mathrm{Bi}_{2} \mathrm{O}_{3}$ is one of the most promising materials which can be used as solid electrolyte [8] in high technology equipment such as fuel cells, sensors, and membrane devices which utilize the unusual high oxide ion conductivity of this compound, a conductivity which exceeds that of stabilized zirconia $[9,10]$. Several structural models for $\delta-\mathrm{Bi}_{2} \mathrm{O}_{3}$ have been proposed [8,11-13]. According to a model by Sillen [12], six possible oxygen sites of the fluorite unit cell are occupied and two others are empty and ordered 

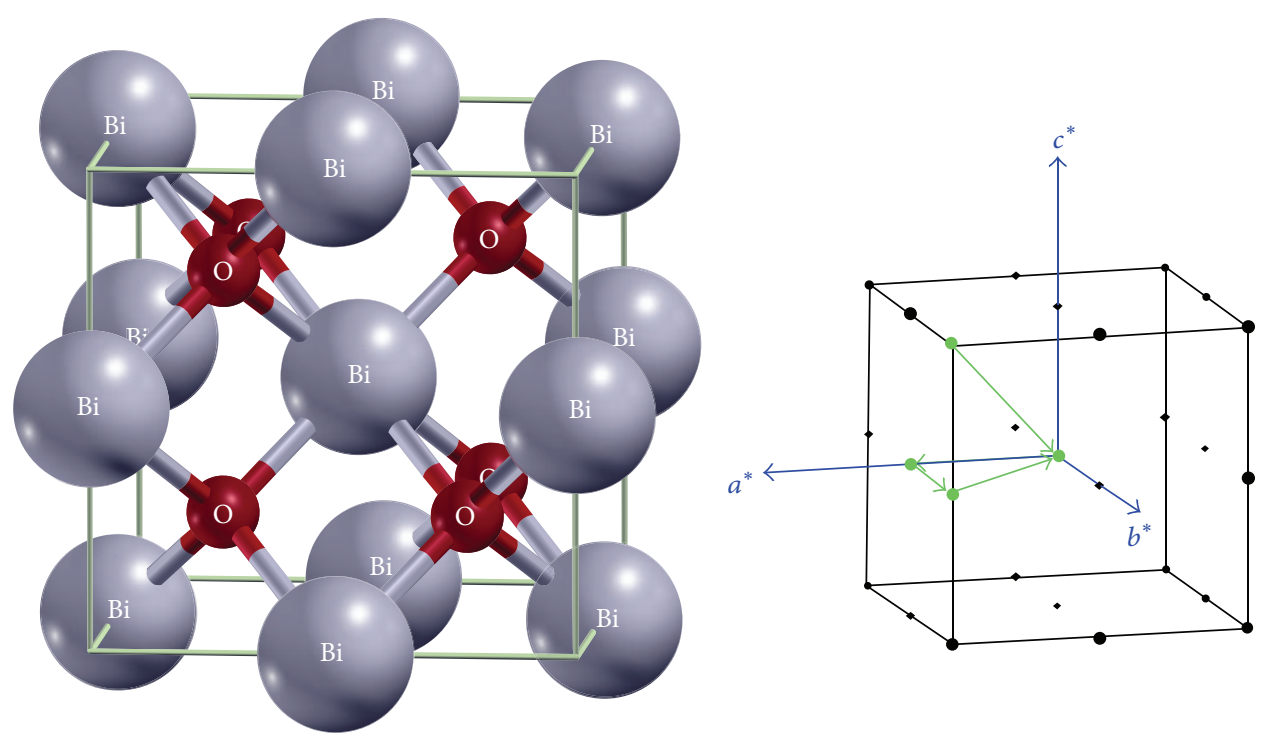

FIGURE 1: Unit cell for $\delta$ - $\mathrm{Bi}_{2} \mathrm{O}_{3}$, with distinct atomic species explicitly indicated. In the inset we show the associated Brillouin zone and the followed path in the energy bands calculation.

in the $\left\langle\begin{array}{lll}1 & 1 & 1\end{array}\right\rangle$ direction. However, the large magnitude of the conductivity suggests that a high disorder on the oxygen sublattice might exist which led other researchers [13] to propose a model of statistically average occupation of all eight oxygen positions.

A number of theoretical studies have been carried out in order to understand the basic properties of metal oxide systems. For example, using the LMTO method, Medvedeva et al. [14] calculated electronic properties of $\delta-\mathrm{Bi}_{2} \mathrm{O}_{3}$ with several oxygen vacancy configurations to find out the role of $\mathrm{Bi}-\mathrm{O}$ and $\mathrm{Bi}-\mathrm{Bi}$ bonding in the stabilization of such derived structures. Several reports have been published concerning $E_{g}$ (width of the forbidden gap between VB and $\mathrm{CB}$ ) values for similar oxides. Gubanov and Medvedeva [15] performed an LMTO-ASA calculation, obtaining values ranging from 1.75 to $5.24 \mathrm{eV}$, respectively, for $\mathrm{SnO}_{2}$ and $\mathrm{ZrO}_{2}$. Different phases of $\mathrm{Bi}_{2} \mathrm{O}_{3}$ like $\alpha-\mathrm{Bi}_{2} \mathrm{O}_{3}, \beta-\mathrm{Bi}_{2} \mathrm{O}_{3}$, and $\delta$ $\mathrm{Bi}_{2} \mathrm{O}_{3}$ were calculated by Evarestov et al. [16] performing a SC-CNDO (Complete Neglect of Differential Overlap) calculation obtaining values ranging from 4.8 to $6.2 \mathrm{eV}$ for the different phases. Reyes [17] using extended Hückel type calculations obtained $3.48 \mathrm{eV}$ in the theoretical study of $\alpha$ $\mathrm{Bi}_{2} \mathrm{O}_{3}$. Walsh et al. [18] reported a theoretical and experimental investigation of the electronic properties of $\delta-\mathrm{Bi}_{2} \mathrm{O}_{3}$ using a combination of gradient corrected density functional theory along with X-ray photoemission and O-K shell X-ray absorption and emission spectroscopy. They reported that vacancies along $\left\langle\begin{array}{lll}1 & 0 & 0\end{array}\right\rangle$ result in the most energetically stable configuration of $\delta$ phase.

Despite the existence of an important number of theoretical studies using DFT, a systematic study to determine what is the most suitable exchange-correlation (XC) potential for this type of metal oxides has not yet been reported. The best performance of GGA schemes over the LDA one is usually claimed, attributing discrepancies to the analytic form of functionals. The aim of this work is to make a detailed comparative study of the structural and electronic properties of $\delta-\mathrm{Bi}_{2} \mathrm{O}_{3}$ in order to assess to what extent the density functional differences are important in the qualitative analysis of theoretical results under the DFT scheme. Besides the XC potential analysis for this bismuth oxide phase, the spin-orbit (SO) interaction effect on electronic properties is also analyzed. We explore distinctive minigaps obtained in our calculations of electronic band structure which are essentially attributed to the SO effect. Conventional density functional calculations were implemented using the APW + lo method based WIEN2k program package, paying careful attention to the lattice parameter, band structure, and density of states as well as charge density profiles. In our calculations it is apparent that the SO effect is not large enough to modify the metallic nature of $\delta-\mathrm{Bi}_{2} \mathrm{O}_{3}$; however, the opening of minigaps distributed along the conduction band is due to this effect. Specific calculation details are given in Section 2; results and discussion are shown in Section 3. In Section 4 we give our conclusions.

\section{Structural and Computational Details}

The structure of $\delta-\mathrm{Bi}_{2} \mathrm{O}_{3}$ used in the present work is that reported by Sillen [12], which is similar to the cubic fluorite structure space group $\mathrm{Pn}-3 \mathrm{~m}$ (group 224) with four Bi atoms in the following positions: 4 (c): $3 / 43 / 43 / 4 ; 3 / 41 / 41 / 4 ; 1 / 43 / 4$ $1 / 4 ; 1 / 41 / 43 / 4$ and six $\mathrm{O}$ atoms located at the following: $6(\mathrm{~d})$ : $01 / 21 / 2 ; 1 / 201 / 2 ; 1 / 21 / 20 ; 1 / 200 ; 01 / 20 ; 01 / 21 / 2$, with the lattice parameter $a=5.525 \AA$ (see Figure 1).

We performed self-consistent calculation of the electronic and atomic structure for $\delta-\mathrm{Bi}_{2} \mathrm{O}_{3}$ using a relativistic fullpotential method based on the augmented plane waves plus local orbitals (APW + lo) [19] as implemented in the WIEN2k code [20], within the framework of DFT [21-23]. The LDA [21, 22], PBE96 [23], and B3PW91 [24-26] energy functionals were used. The nonequivalency of PW91 and PBE functionals [27] was recently reported, which makes us cautious in the 


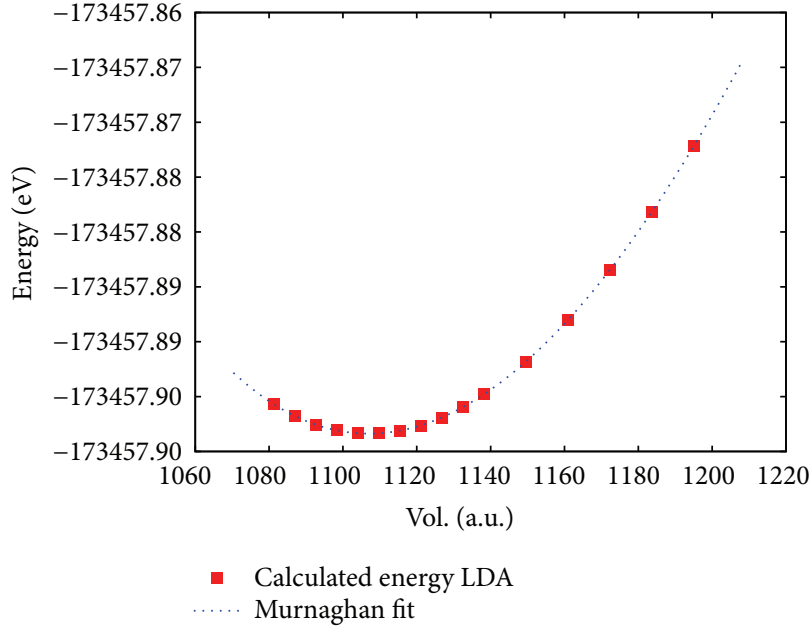

(a)

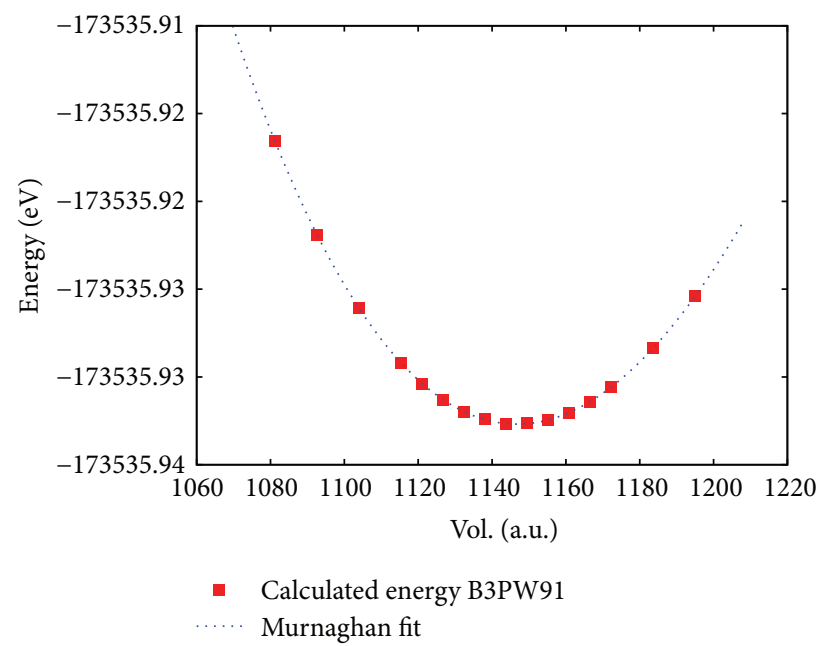

(b)

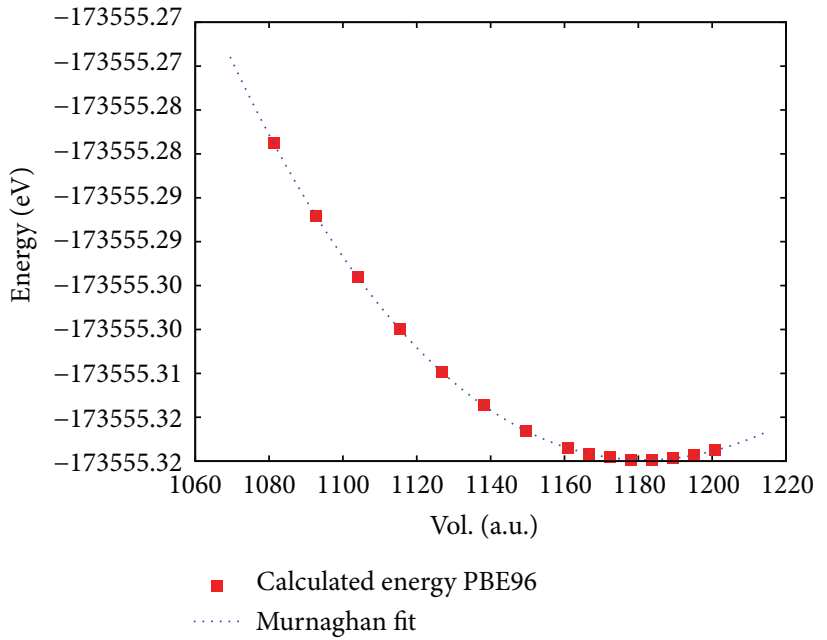

(c)

FIgURE 2: $E_{\text {tot }}$ versus volume curve for $\delta-\mathrm{Bi}_{2} \mathrm{O}_{3}$, obtained with the functionals (a) LDA, (b) B3PW91, and (c) PBE96.

calculation of electronic properties of solid materials even though PBE is yet considered the standard approach to determine structural properties in solid state physics [28-30]. A comparison of results using LDA, PBE96, and B3PW91 functionals provides a broader insight into the use of density functionals for the prediction of physical and chemical properties in different bismuth oxide phases. It is worth mentioning that the hybrid B3PW91 functional includes $20 \%$ of Hartree-Fock exchange, which is not considered in LDA or PBE96.

In our calculations, the muffin-tin sphere radii were fixed at $R_{\mathrm{mt}}=1.8$ a.u. for oxygen atoms and $R_{\mathrm{mt}}=2.3 \mathrm{a}$.u. for bismuth atoms. The maximum $l$ value for partial waves used inside atomic spheres was $l_{\max }=10$, while the magnitude of the largest vector in charge density Fourier expansion is fixed at $G_{\max }=14$. The basis set includes $2 \mathrm{~s}^{2} 2 \mathrm{p}^{4}$ of oxygen and $5 \mathrm{~d}^{10} 6 \mathrm{~s}^{2} 6 \mathrm{p}^{3}$ of bismuth as valence states, because the electronic charge of these states is not contained completely within the muffin-tin spheres. In this computational code, the relativistic effects for core states are taken into account using a scalar-relativistic basis, while the spin-orbit coupling is calculated using the second-variation method [31-33].
The band structure of $\delta-\mathrm{Bi}_{2} \mathrm{O}_{3}$ is calculated using $10 \times 10 \times$ $10 k$-point mesh in the first Brillouin zone (FBZ) for the SCF cycle, plotting the energy versus $k$-point values following high symmetry points from $(1 / 2,1 / 2,1 / 2)$ to $(0,0,0)$ to $(1 / 2,0,0)$ to $(1 / 2,1 / 2,0)$ to $(0,0,0)$ of the value of $\pi / a$ to span the threedimensional $k$ space (see inset in Figure 1 ). The density of states was computed using $20 \times 20 \times 20 k$-point mesh while the Fermi energy $\left(E_{F}\right)$ and the weights of each energy band were calculated using a temperature broadening scheme with a value of 0.005 . For both structural and electronic properties calculation we used a plane wave cut-off of $R_{\mathrm{mt}} K_{\max }=7.5$.

\section{Results and Discussion}

3.1. Structural Properties. Structural properties were calculated from the ground state as a function of volume. A uniform compression and expansion of the lattice with the relative atomic positions within the unit cell held constant were performed to make an isotropic variation of the cell volume, thus searching for the stable crystalline structure. The calculated total energy for $\delta-\mathrm{Bi}_{2} \mathrm{O}_{3}$ is displayed as a function of the cell volume in Figures 2(a)-2(c). The total 


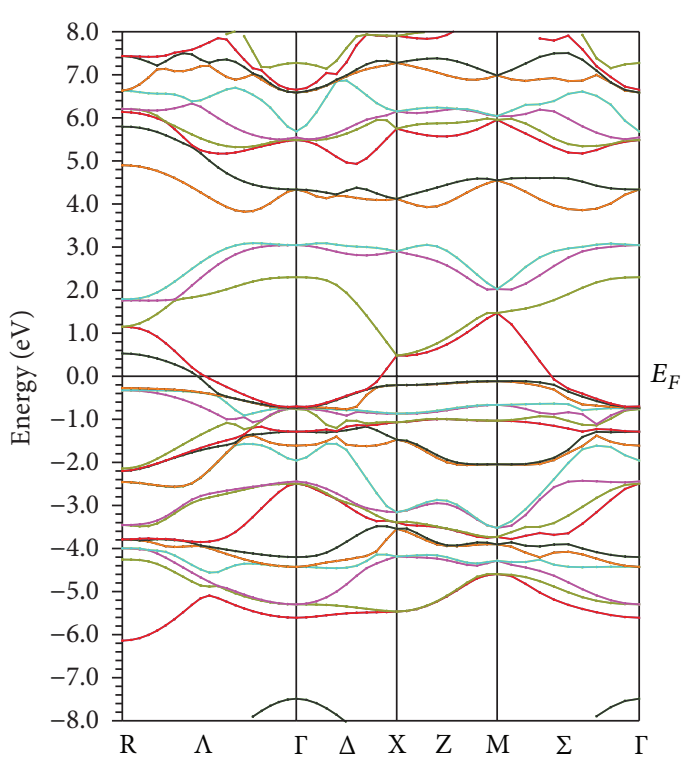

(a)

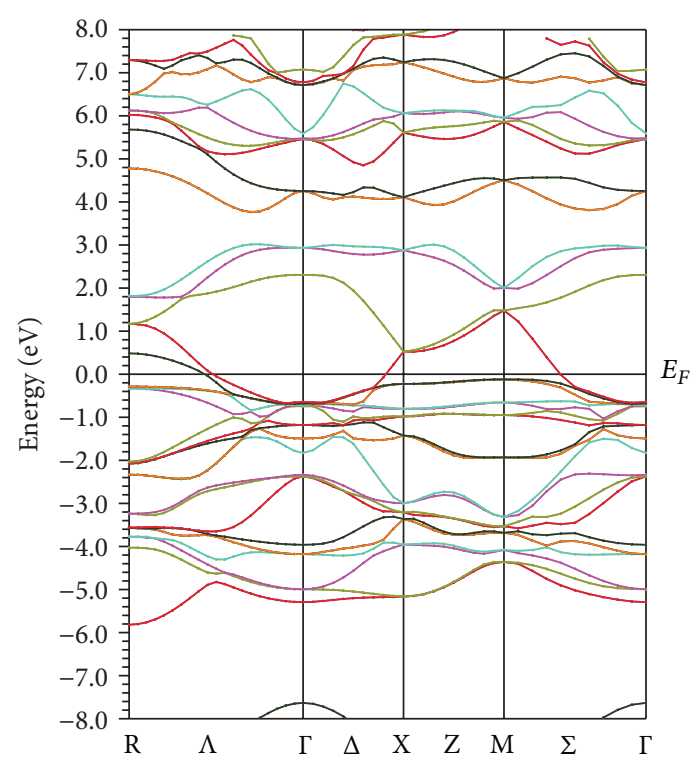

(b)

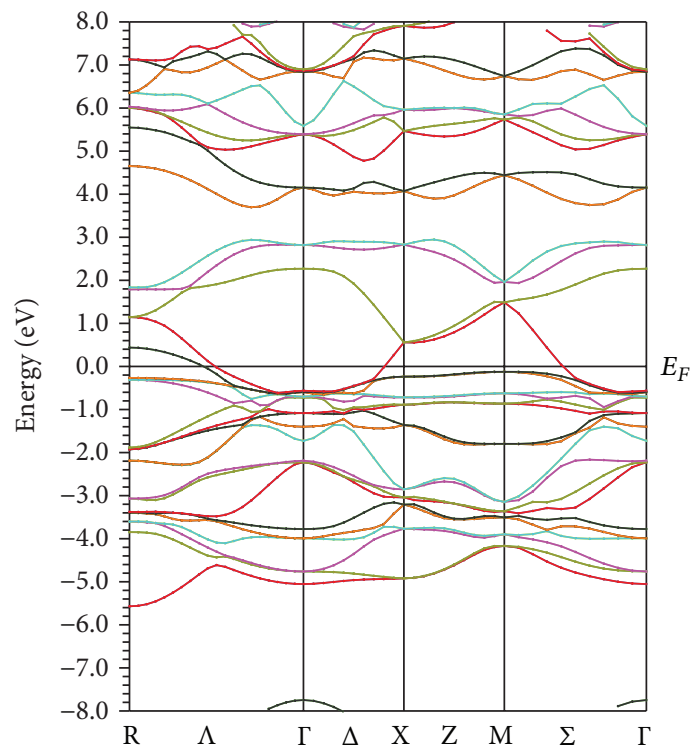

(c)

FIGURE 3: Calculated energy bands for $\delta$ - $\mathrm{Bi}_{2} \mathrm{O}_{3}$ considering spin-orbit interaction and using density functionals (a) LDA, (b) B3PW91, and (c) PBE96. Fermi energy $\left(E_{F}\right)$ is located at $0.0 \mathrm{eV}$.

energy values were used to fit the Murnaghan [34] equation of state from which we obtained the optimized lattice parameter and the bulk modulus. Notice that an exceptional fit was achieved for the cell expansion in the three studied cases.

To obtain the equilibrium unit cell, total energy versus volume (see Figure 2) was calculated without the spin-orbit interaction using the following parameters: $R_{\mathrm{mt}} K_{\max }=7.5$, initial experimental parameter $a=5.525 \AA$, and $10 \times 10$ $\times 10 k$-points sampling the first Brillouin zone (FBZ). This procedure yielded the equilibrium lattice parameters showed in Table 1. Unit cell optimization taking into account the spinorbit interaction was also performed using the PBE96 functional, through the same procedure to understand whether it is significant or instead it is irrelevant in the calculation of the lattice parameter. The difference between both values is of the order of $0.01 \AA$; that is, there is a very small effect due to spin-orbit (SO) interaction, and thus the calculations of the electronic properties were made using the calculated equilibrium lattice parameters without SO effect. The lattice parameter calculated using the B3PW91 hybrid functional is in better agreement with the experimental value $a=5.525 \AA$.

3.2. Electronic Properties. Figures 3(a)-3(c) display energy bands versus $k$-points for $\delta-\mathrm{Bi}_{2} \mathrm{O}_{3}$, considering spin-orbit interaction. By examining the electronic band structure, we notice that the band shape is similar and that the Fermi level $\left(E_{F}\right)$ is crossed by multiple degenerate bands indicating a metallic behavior, regardless of the XC functional. On the other hand, the addition of SO coupling splits orbital degeneracies into some high symmetry points in the FBZ. 

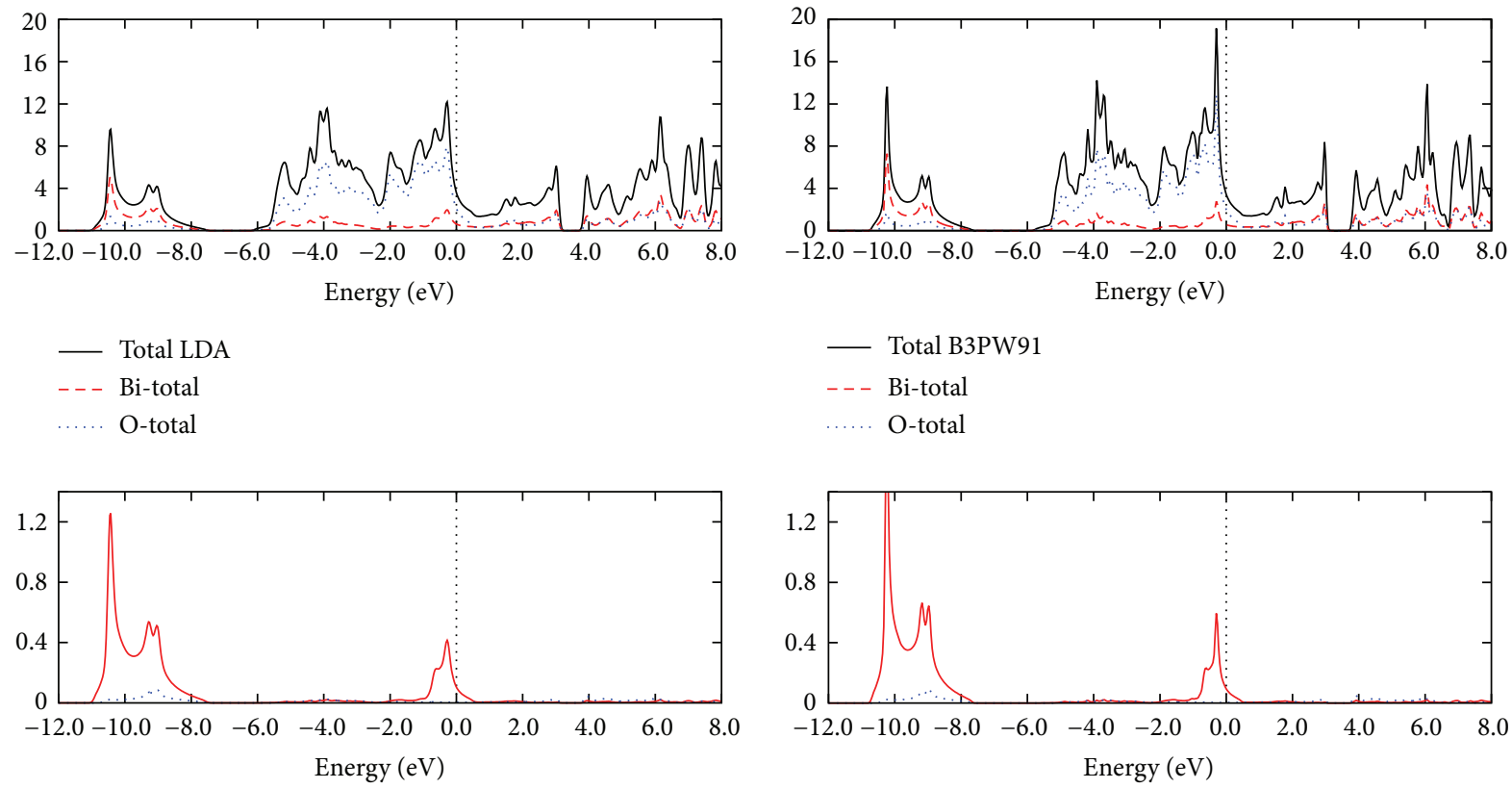

— Bi-s

- Bi-s

$\mathrm{O}-\mathrm{s}$

O-s
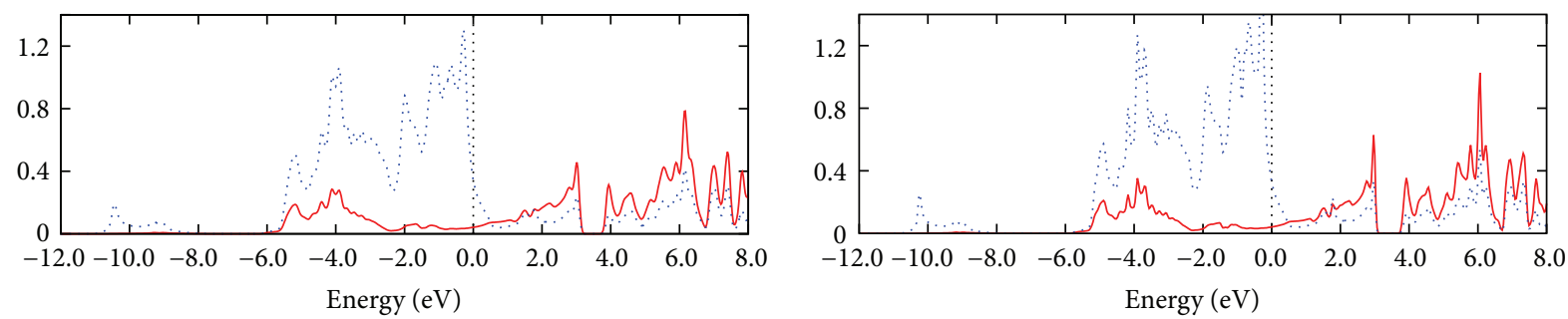

— Bi-p

— Bi-p

… O-p

…. O-p
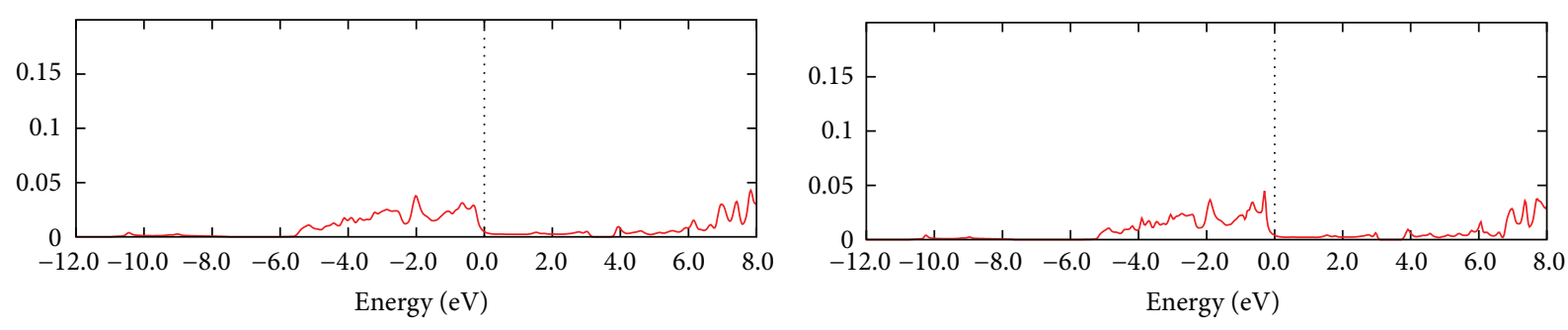

— Bi-d

— Bi-d
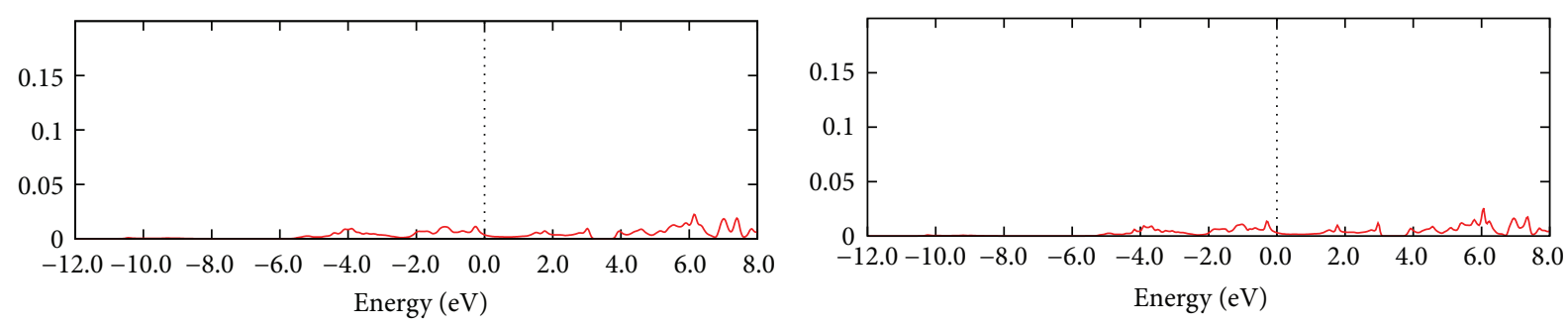

— Bi-f

— Bi-f

(a)

(b)

FIGURE 4: Continued. 

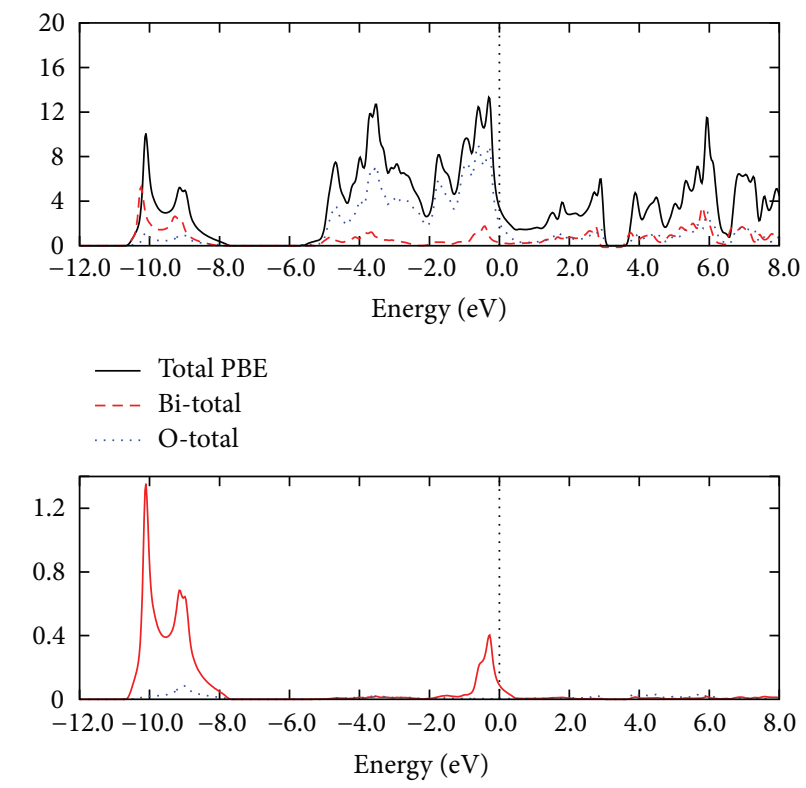

- Bi-s

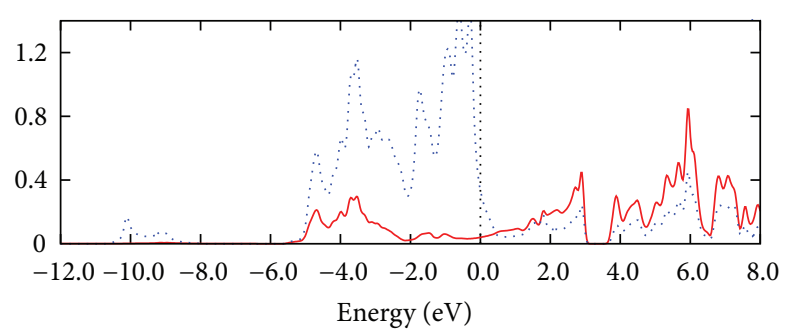

- Bi-p

…. O-p

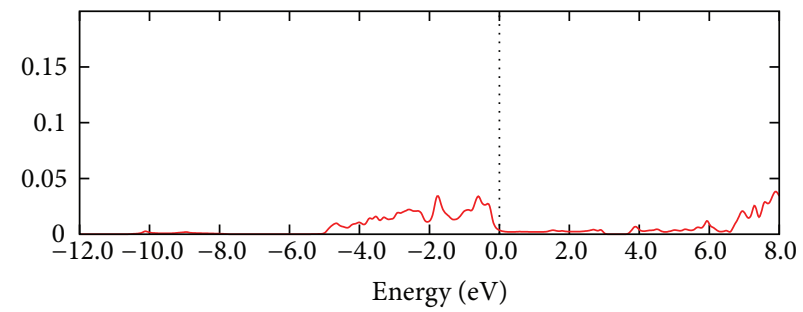

- Bi-d

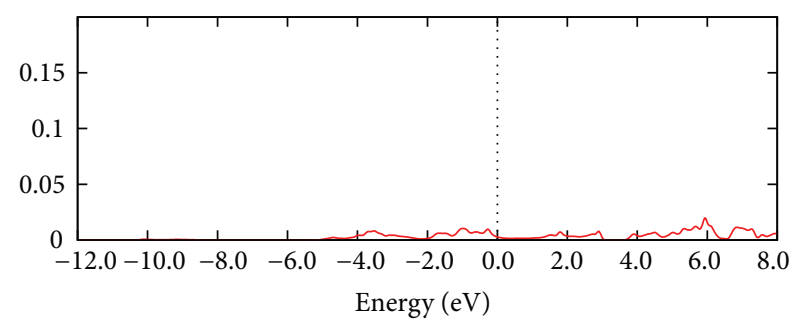

- Bi-f

(c)

FIGURE 4: Total and projected density of states (DOS) for $\delta-\mathrm{Bi}_{2} \mathrm{O}_{3}$ for the case when spin-orbit interaction is considered and using functionals (a) LDA, (b) B3PW91, and (c) PBE96. 


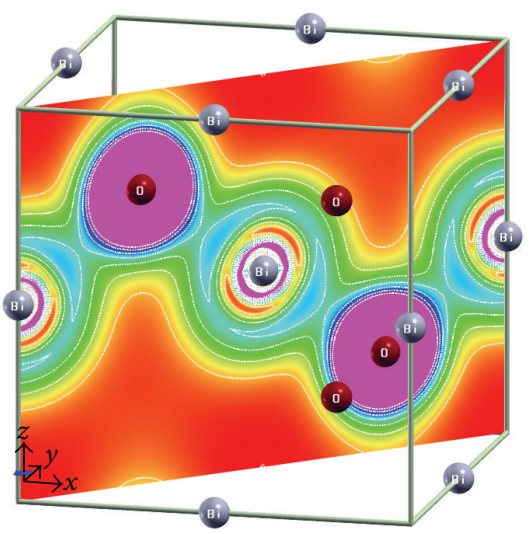

Electron density

$$
\begin{array}{ll}
\square+0.0125 & \square+0.3050 \\
\square+0.1100 & \square+0.4025 \\
\square+0.2075 & \square+0.5000
\end{array}
$$

(a)

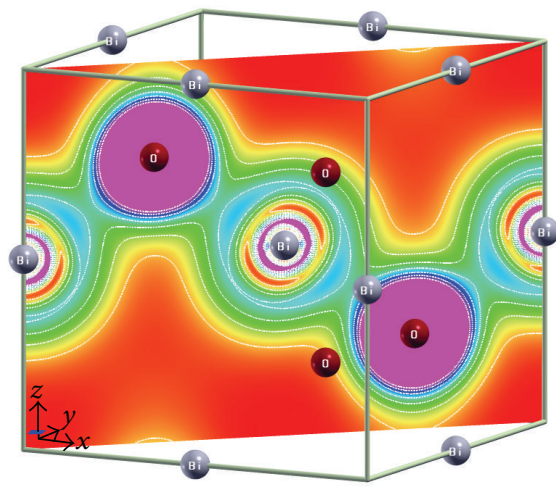

Electron density

$\square+0.0109$

$\square+0.1087$

ㅁ +0.2066

$\square+0.3044$

+0.4022

口 +0.5000

(c)

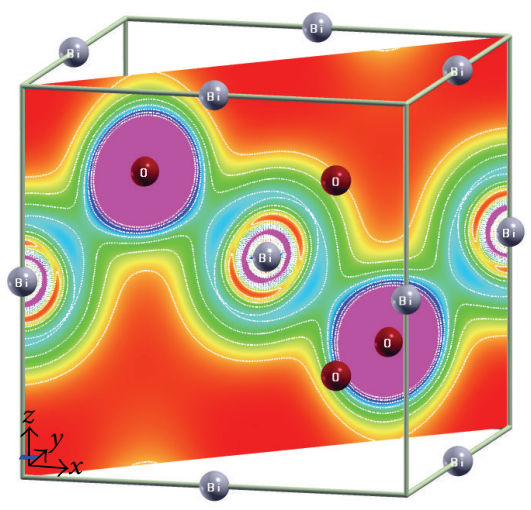

Electron density

+0.0099

$\square+0.1079$

$\square+0.2059$

$\square+0.3040$
घ +0.4020

(e)

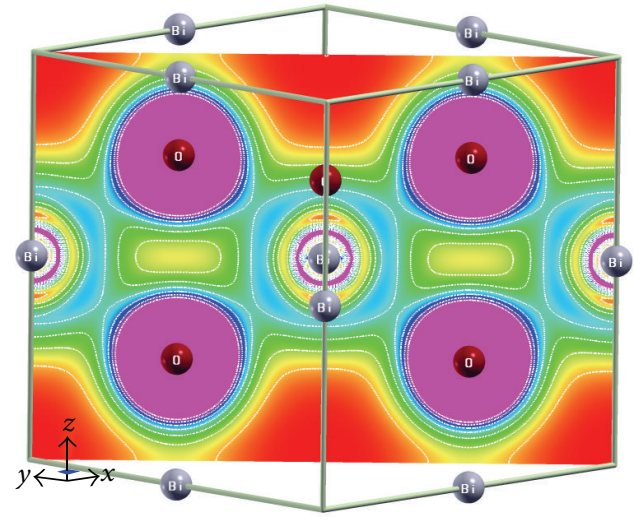

Electron density
ㅁ +0.0125
$\square+0.3050$
$\square+0.1100$
$\square+0.4025$
$\square+0.2075$
$\square+0.5000$

(b)

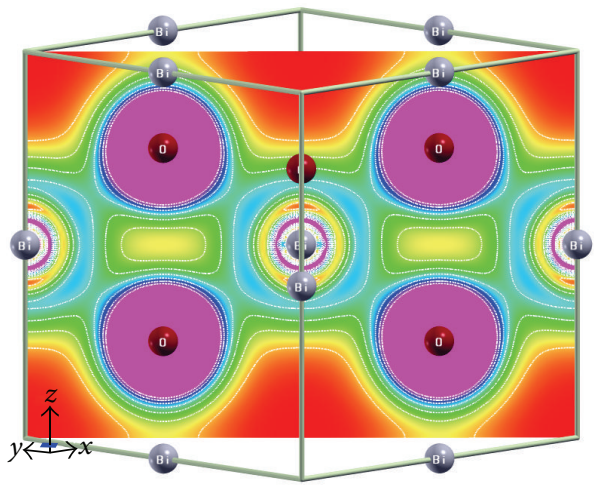

Electron density
$\square+0.0109$
$\square+0.3044$
ㅁ +0.1087
口 +0.4022
口+0.2066
口 +0.5000

(d)

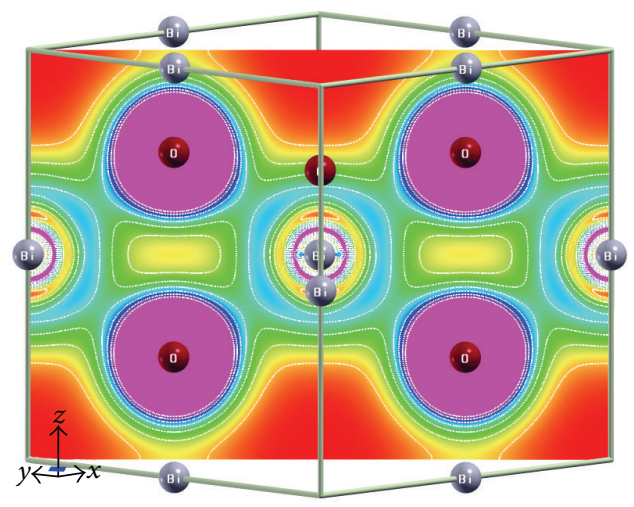

Electron density
ㅁ +0.0099
$\square+0.3040$
$\square+0.1079$
口 +0.4020
ㅁ +0.2059
口 +0.5000

(f)

FIGURE 5: Charge density profile for $\delta$ - $\mathrm{Bi}_{2} \mathrm{O}_{3}$ obtained by using LDA (a, b), B3PW91 (c, d), and PBE96 (e, f) exchange-correlation functionals. The charge profiles are plotted in (100) and (110) planes. Each atom has been identified with the corresponding chemical symbol. 
TABLE 1: Lattice parameters for $\delta-\mathrm{Bi}_{2} \mathrm{O}_{3}$ calculated in this work. The experimental value ${ }^{\mathrm{a}}$ is $5.525 \AA$.

\begin{tabular}{lc}
\hline XC functional & $a(\AA)$ without SO \\
\hline LDA & 5.4742 \\
B3PW91 & 5.5389 \\
PBE96 & 5.5946 \\
\hline
\end{tabular}

${ }^{\mathrm{a}}[15]$.

Figure 3 exhibits three distinctive groups of energy bands showing a band gap between them. The first group is below $-7.5 \mathrm{eV}$, a second group is between -5.6 and $2.9 \mathrm{eV}$, crossing the Fermi level, and the third group is above $3.6 \mathrm{eV}$.

The total and projected density of states (DOS) for $\delta$ $\mathrm{Bi}_{2} \mathrm{O}_{3}, \mathrm{Bi}$, and $\mathrm{O}$ atoms, considering spin-orbit interaction, are provided in Figures 4(a)-4(c) where energy versus DOS is plotted. Notice that the $\mathrm{d}$ and $\mathrm{f}$ contributions for $\mathrm{Bi}$ are not present in the DOS graphs but are taken into account on its shape because they are included in the relativistic approach. Analysis of the band structure and partial DOS permits us to gain insight into their electronic (orbital) origin. The main contribution to the band from -8.0 to $-10.0 \mathrm{eV}$ comes from Bi $6 s$, identified as the bulk response given by the electrons closer to the nucleus. The following band -6.0 to $+3.0 \mathrm{eV}$ is formed by $\mathrm{Bi} 6 \mathrm{~s}(0.4$ states/eV) and $\mathrm{Bi} 6 \mathrm{p}(0.2-0.4$ states/eV) orbitals, while $\mathrm{O} 2 \mathrm{p}$ orbital contributes with 1.4 states/eV. The Fermi level was shifted to $0.0 \mathrm{eV}$ to perform an analysis of the contributions from each atom in the vicinity of $E_{F}$. According to the orbital analysis, it is mainly formed by hybridized $\mathrm{Bi} 6 \mathrm{~s}$ ( 0.4 states/eV) and $\mathrm{O} 2 \mathrm{p}(1.4$ states/eV) orbitals. Finally, the conduction bands from +3.5 to $6.0 \mathrm{eV}$ are mainly formed by Bi $6 \mathrm{p}(0.2-1.0$ states/eV) contribution.

To test the degree of covalency or ionicity in $\mathrm{Bi}_{2} \mathrm{O}_{3}$, electronegativity values (in the Pauling scale) of $\mathrm{Bi}(2.02)$ and $\mathrm{O}$ (3.44) give us a clue on the bonding character. Thus, based on the Pauling electronegativity scale [35], the electronegativity difference is about 1.42 which indicates to us that the amount of ionic character is $\sim 40 \%$. This suggests that the ionic character is significant in the bond between $\mathrm{Bi}$ and $\mathrm{O}$ but the covalent character is greater, and therefore we expect to find a fraction of electron density shared in-between. In order to verify the predicted ionic contribution on bonding type we carried out valence charge density calculations whose contours for $\delta-\mathrm{Bi}_{2} \mathrm{O}_{3}$ are plotted in (100) and (110) planes, depicted in Figures 5(a)-5(f), where each atom has been labeled by its chemical symbol. These figures exhibit a similar charge distribution for the different XC potentials used and the existence of almost uniform charge distribution between $\mathrm{Bi}$ and $\mathrm{O}$, which provides additional evidence of the ionic bonding feature of this compound. A quantitative analysis of the charge density between $\mathrm{Bi}$ and $\mathrm{O}$ atoms shows that the charge density is, at least, of $0.2 \mathrm{e} / \AA^{3}$. All calculations were carried out considering spin-orbit interaction effects.

\section{Concluding Remarks}

The structural and electronic properties of $\delta-\mathrm{Bi}_{2} \mathrm{O}_{3}$ were calculated using WIEN2K employing relativistic considerations for $\mathrm{Bi}$ and $\mathrm{O}$ atoms and the exchange-correlation functionals LDA, PBE96, and the hybrid B3PW91. Structural optimization yielded an equilibrium lattice parameter of $a_{\mathrm{LDA}}=$ $5.4742 \AA, a_{\mathrm{B} 3 \mathrm{PW} 91}=5.5389 \AA$, and $a_{\mathrm{PBE} 96}=5.5946 \AA$. The overestimation using PBE96 and underestimation using LDA with regard to the experimental value $(a=5.525 \AA)$ are within the expected on a GGA and LDA approach, respectively. A meticulous analysis of electronic properties was also performed. The energy bands were identified to have $\mathrm{s}$ and $\mathrm{p}$ character. Our results also provide evidence that the system behaves like a metal, considering the spinorbit interaction. The major contributions to each band were identified from the projected DOS. The bands closer to $E_{F}$ $(0$ to $-1.0 \mathrm{eV})$ are formed mainly from $\mathrm{O} 2 \mathrm{p}(1.4$ states/eV) and $\mathrm{Bi} 6 \mathrm{~s}$ (1.5 states/eV) hybridized orbitals. Finally, the charge density profiles exhibit the partial ionic chemical bonding features of this compound, with a small amount of charge between $\mathrm{Bi}$ and $\mathrm{O}$ atoms as a consequence of the partial covalent bonding. Even though most of the electronic properties describe similar behavior for each of the XC functionals used, the lattice parameter obtained with the B3PW91 functional is in better agreement with the experimental value, probably due to the existence of Hartree-Fock exchange in this functional.

\section{Conflict of Interests}

The authors declare that there is no conflict of interests regarding the publication of this paper.

\section{Acknowledgments}

D. H. Galván acknowledges CONACYT under Grant 3027PE and Proyecto de Supercomputo SC-004696 for the support provided. R. Núñez-González acknowledges Área de Cómputo de Alto Rendimiento (ACARUS) at Universidad de Sonora for giving access to their supercomputer system. A. Posada-Amarillas is grateful to Dr. L. Oliver Paz-Borbón from Chalmers University of Technology for useful discussions and valuable suggestions throughout this investigation.

\section{References}

[1] A. F. Ioffe, Poluprovodnikovie Termoelementary, Izvestiya Akademii Nauk SSSR, Moskova, Russia, 1960 (Russian).

[2] J. M. Thomas, D. A. Jefferson, and G. R. Willward, "The nanostructure of heterogeneous catalysts," JEOL News E, vol. 23, no. 7, p. 7, 1985.

[3] D. H. Galván, S. Fuentes, M. Avalos-Borja et al., "Structure and catalytic activity characterization of bismuth molybdate catalysts," Catalysis Letters, vol. 18, no. 3, pp. 273-281, 1993.

[4] K. Fossheim, E. D. Tuset, T. W. Ebbesen, M. M. J. Treacy, and J. Schwartz, "Enhanced flux pinning in $\mathrm{Bi}_{2} \mathrm{Sr}_{2} \mathrm{CaCu}_{2} \mathrm{O}_{8+x}$ superconductor with embedded carbon nanotubes," Physica C: Superconductivity, vol. 248, no. 3-4, pp. 195-202, 1995.

[5] A. J. Salazar-Pérez, M. A. Camacho-López, R. A. MoralesLuckie, V. Sánchez-Mendieta, F. Ureña-Ñúñez, and J. ArenasAlatorre, "Structural evolution of $\mathrm{Bi}_{2} \mathrm{O}_{3}$ prepared by thermal oxidation of bismuth nano-particles," Superficies y vacío, vol. 18, pp. 4-8, 2005. 
[6] B. Y. Liaw and W. Weppner, "Low temperature limiting-current oxygen sensors based on tetragonal zirconia polycrystals," Journal of the Electrochemical Society, vol. 138, no. 8, pp. 24782483, 1991.

[7] N. Cornei, N. Tancret, F. Abraham, and O. Mentré, "New $\mathcal{\varepsilon}$ $\mathrm{Bi}_{2} \mathrm{O}_{3}$ metastable polymorph," Inorganic Chemistry, vol. 45, no. 13, pp. 4886-4888, 2006.

[8] E. C. Subbarao and H. S. Maiti, "Solid electrolytes with oxygen ion conduction,” Solid State Ionics, vol. 11, no. 4, pp. 317-338, 1984.

[9] V. P. Zhuk, A. A. Vecher, and V. V. Samokhval, Vestn. Beloruss. Gos. Univ., vol. 2, no. 1, pp. 8-15, 1984.

[10] T. Takahashi, H. Iwahara, and T. Arao, "High oxide ion conduction in sintered oxides of the system $\mathrm{Bi}_{2} \mathrm{O}_{3}-\mathrm{Y}_{2} \mathrm{O}_{3}$," Journal of Applied Electrochemistry, vol. 5, no. 3, pp. 187-195, 1975.

[11] H. A. Harwig and Z. Anorg, "On the structure of bismuthsesquioxide: the $\alpha, \beta, \gamma$, and $\delta$-phase," Zeitschrift für Anorganische und Allgemeine Chemie, vol. 444, no. 1, pp. 151-166, 1978.

[12] L. G. Sillén, "X-ray studies on Bismuth-Trioxili," Arkiv för Kemi, Mineralogi och Geologi A, vol. 12, no. 18, pp. 1-15, 1937.

[13] G. Gattow and H. Schröder, "Über wismutoxide. III. Die kristallstruktur der hochtemperaturmodifikation von Wismut(III)-oxid $\left(\delta-\mathrm{Bi}_{2} \mathrm{O}_{3}\right)$, , Zeitschrift für Anorganische und Allgemeine Chemie, vol. 318, no. 3-4, pp. 176-189, 1962.

[14] N. I. Medvedeva, V. P. Zhukov, V. A. Gubanov, D. L. Novikov, and B. M. Klein, "Electronic structure and chemical bonding in $\delta-\mathrm{Bi}_{2} \mathrm{O}_{3}$," Journal of Physics and Chemistry of Solids, vol. 57, no. 9, pp. 1243-1250, 1996.

[15] V. A. Gubanov and N. I. Medvedeva, "Electronic band structure and chemical bonding in the transition metal dioxides," Physica B: Condensed Matter, vol. 172, no. 1-2, pp. 285-288, 1991.

[16] R. A. Evarestov, V. O. Shapovalov, and V. A. Veryazov, "Electronic structure and chemical bonding in $\mathrm{Bi}_{2} \mathrm{O}_{3}$," Physica Status Solidi (b), vol. 183, no. 1, pp. K15-K17, 1994.

[17] J. A. Samaniego-Reyna, Estructura electronic del $\alpha-B i_{2} \mathrm{O}_{3}$ con el metodo de enlace-fuerte Hückel Extendido [M.S. thesis], CICESE, Ensenada, Mexico, 1997.

[18] A. Walsh, G. W. Watson, D. J. Payne et al., "Electronic structure of the $\alpha$ and $\delta$ phases of $\mathrm{Bi}_{2} \mathrm{O}_{3}$ : a combined ab initio and $\mathrm{x}$-ray spectroscopy study," Physical Review B, vol. 73, no. 23, Article ID 235104, 2006.

[19] G. K. H. Madsen, P. Blaha, K. Schwarz, E. Sjöstedt, and L. Nordström, "Efficient linearization of the augmented planewave method," Physical Review B, vol. 64, Article ID 195134, 2001.

[20] P. Blaha, K. Schwarz, G. K. H. Madsen, D. Kvasnicka, and J. Luiz, WIEN2K, An Augumented Plane Wave + Local Orbital Program for Calculating Crystal Properties, WIEN2K 09.2, Vienna University of Technology, Vienna, Austria, 2009.

[21] P. Hohenberg and W. Kohn, "Inhomogeneous electron gas," Physical Review, vol. 136, article B864, 1964.

[22] W. Kohn and L. J. Sham, "Self-consistent equations including exchange and correlation effects," Physical Review, vol. 140, no. 4, pp. A1133-A1138, 1965.

[23] J. P. Perdew, K. Burke, and M. Ernzerhof, "Generalized gradient approximation made simple," Physical Review Letters, vol. 77, no. 18 , article $3865,1996$.

[24] A. P. Scott and L. Radom, "Harmonic vibrational frequencies: an evaluation of Hartree-Fock, Møller-Plesset, quadratic configuration interaction, density functional theory, and semiempirical scale factors," Journal of Physical Chemistry, vol. 100, no. 41, pp. 16502-16513, 1996.
[25] P. Geerlings, F. De Proft, and J. M. L. Martin, Theoretical and Computational Chemistry, vol. 4 of Recent Developments and Applications of Modern Density Functional Theory, Elsevier, New York, NY, USA, 1996, edited by: J. Seminario.

[26] D. Torumba, P. Novák, and S. Cottenier, "Hybrid exchangecorrelation functionals applied to hyperfine interactions at lanthanide and actinide impurities in Fe," Physical Review B, vol. 77, no. 15, Article ID 155101, 2008.

[27] A. E. Mattsson, R. Armiento, P. A. Schultz, and T. R. Mattsson, "Nonequivalence of the generalized gradient approximations PBE and PW91," Physical Review B-Condensed Matter and Materials Physics, vol. 73, no. 19, Article ID 195123, 2006.

[28] L. S. Pedroza, A. J. R. da Silva, and K. Capelle, "Gradientdependent density functional of the Perdew-Burke-Ernzenhof type for atoms, molecules and solids," Physical Review B, vol. 79, no. 20, Article ID 201106, 4 pages, 2009.

[29] P. Hass, F. Tran, P. Blaha, K. Schwarz, and R. Laskowski, "Insight into the performance of GGA functionals for solidstate calculations," Physical Review B, vol. 80, no. 19, Article ID 195109, 13 pages, 2009.

[30] P. Haas, F. Tran, P. Blaha et al., "Systematic investigation of a family of gradient-dependent functionals for solids," Physical Review B-Condensed Matter and Materials Physics, vol. 81, no. 12, Article ID 125136, 2010.

[31] D. D. Koelling and B. N. Harmon, "A technique for relativistic spin-polarised calculations," Journal of Physics C: Solid State Physics, vol. 10, no. 16, article 3107, 1977.

[32] A. H. MacDonald, W. E. Picket, and D. D. Koelling, "A linearised relativistic augmented-plane-wave method utilising approximate pure spin basis functions," Journal of Physics C: Solid State Physics, vol. 13, no. 14, article 2675, 1980.

[33] D. Sing, Plane Waves, Pseudopotentials and the LAPW Method, Kluwer Academic Publishers, 1994.

[34] F. D. Murnaghan, "The compressibility of media under extreme pressures," Proceedings of the National Academy of Sciences of the United States of America, vol. 30, pp. 244-247, 1944.

[35] L. Pauling, General Chemistry, chapter 6, Dover Publications, New York, NY, USA, 3rd edition, 1988. 

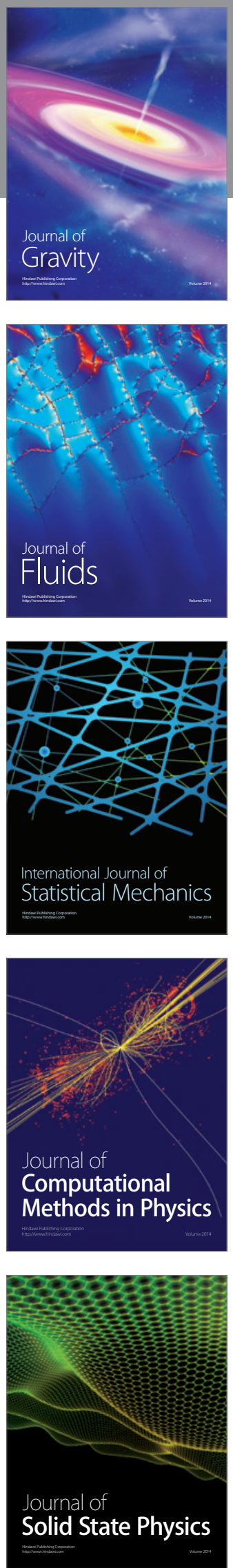

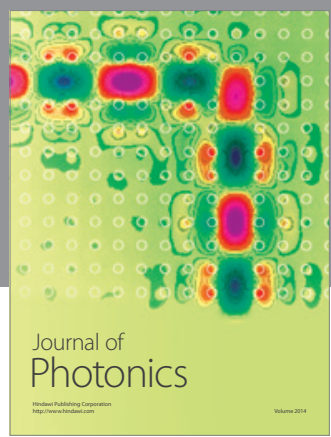

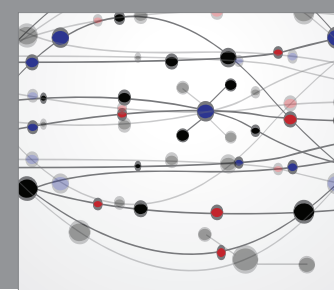

The Scientific World Journal

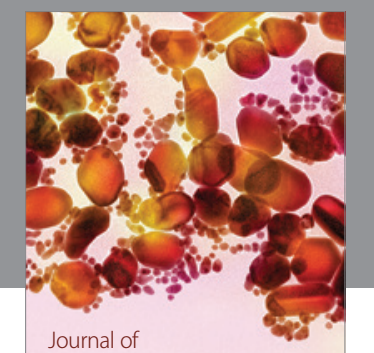

Soft Matter
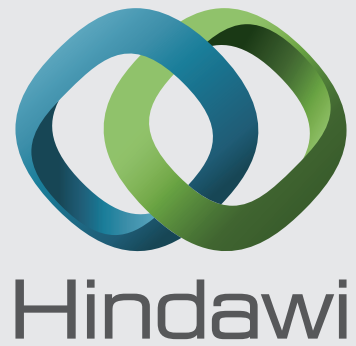

Submit your manuscripts at

http://www.hindawi.com
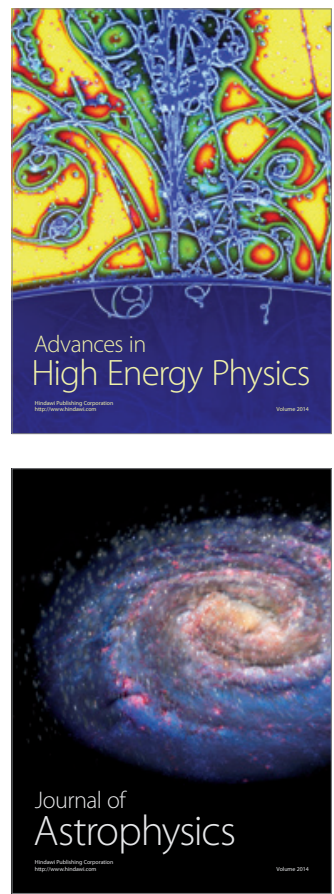
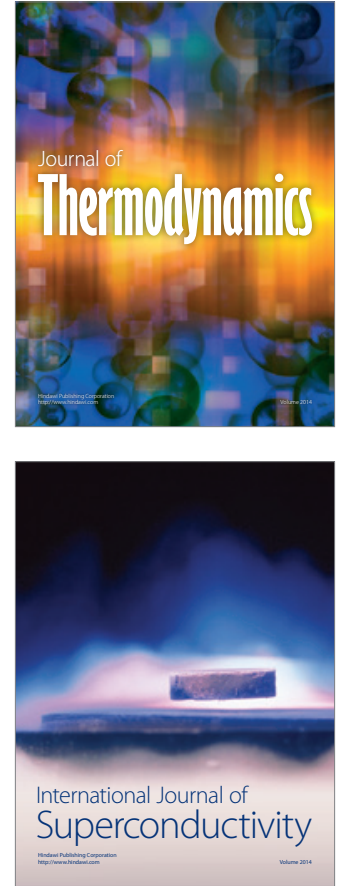
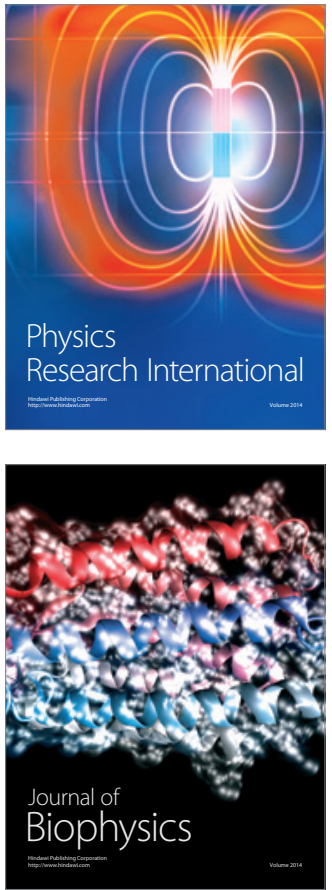
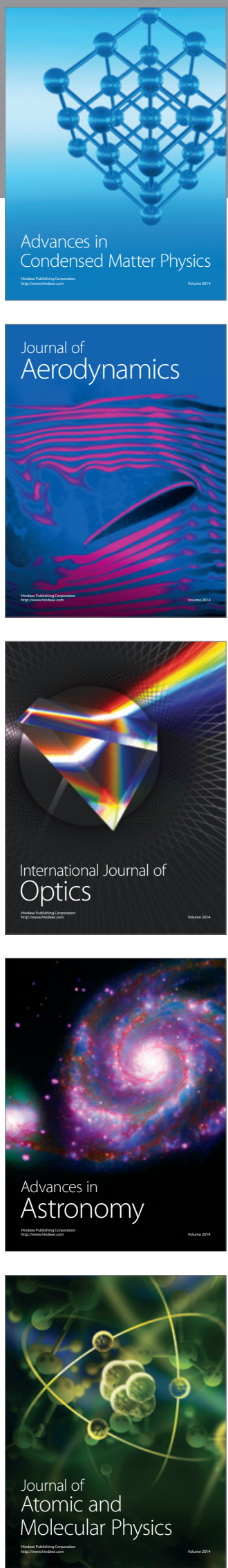